\title{
A Two-Patch Ecological System with Nonlinear Transfer Rate and Noise Effect
}

\author{
Suqi Ma, Zhaosheng Feng, and Qishao Lu \\ Communicated by $Y$. Charles Li, received June 14, 2008.
}

\begin{abstract}
In this paper, we study the dynamical behavior of a species which inhabits two independent habitat patches. Due to the long range foraging behavior, frequent transfers happen between two patches with an exponentially decaying nonlinear transfer rate. Periodic oscillation is observed as a Hopf bifurcation occurs at some critical values of the delay $\tau$. By applying the center manifold theorem, the Poincaré normal form and the approximate periodic solution near the critical delay values are obtained. The complete synchronization of variations of the population size of species in two patches is analyzed and numerical simulations under various parametric conditions are illustrated. The moment stability of the solution of the stochastic delay equation is also considered by applying the Itô integral.
\end{abstract}

\section{Contents}

1. Introduction

2. Stability Analysis of the Balance State $\left(x^{*}, y^{*}\right)$ Without the Noise Term 284

3. Poincaré Normal Form

4. Moment Stability with the Noise Perturbation 292

5. Conclusion 296

$\begin{array}{ll}\text { Acknowledgments } & 297\end{array}$

$\begin{array}{ll}\text { References } & 297\end{array}$

1991 Mathematics Subject Classification. Primary: 92D40, 37M20; Secondary: 65P30.

Key words and phrases. Delay differential equation, stochastic equation, Poincaré normal form, Hopf bifurcation, center manifold, noise effect, synchronization solution.

Main contents of this paper have been presented at the Third International Symposium on Nonlinear Sciences and Applications, Fudan University, Shanghai, China, June 6-10, 2007. The work is supported by NSF (China) Grant 10432010 and partly supported by CRA (Chinese Agricultural University) Doctorate Grant 2006064. 


\section{Introduction}

As we know, many consumer species go through two or more life stages as they proceed from birth to death. A large number of the models in the existing literature ignore such reality and lump individuals into one single reproducing category which can be modeled by a single ordinary differential equation (ODE) [1]. However, such simple ODEs are only capable of generating simple equilibrium dynamics. In order to capture the oscillatory and chaotic behavior often observed in nature, various mechanisms are proposed. Such mechanisms mainly include difference models and delay differential models [2]. Due to finite propagation speeds of signals, finite processing times in synapses, and finite reaction time, time delay is ubiquitous in many of physical [3], chemical [4, 5], biological [1, 2], neural [6] and other natural phenomena. In the population biology literature, the numbers of biological and ecological population often display a highly dynamic nature characterized by statistically significant oscillations. Theoretical models with time delay are postulated and analyzed in an attempt to explain these oscillations. Mathematically, it becomes very natural and often of interest to ask under what conditions bifurcations, oscillations, and even chaos occur while we take into account the length of time delay.

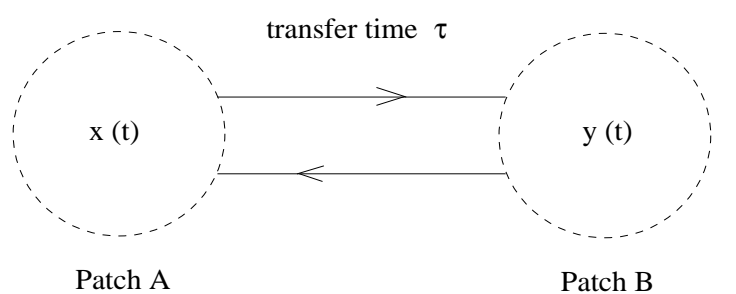

Figure 1. Schematic figure for the species inhabiting two patches and transferring between each other.

In the present paper, we are concerned with the persistence characteristic of a simple species which inhabits two independent patches $A$ and $B$ (see Figure 1). The fact that stochastic perturbations occur in the real world may lead to instability and oscillation. For example, the birth and the death may randomly vary due to the season switching, the change of climate and weather, etc. Therefore, in our study, on one of patches we take into account the effect of random factor such as noise, which may help us better understand the oscillatory behavior of system for the population size of a species. We assume that the species growth is governed by the following ODE with the Allee effect [7]

$$
N^{\prime}(t)=a N(t)\left(1-\alpha_{1} N(t)-\alpha_{2} N^{2}(t)\right)-d N(t)
$$

where $N(t)$ denotes the population density of one species, and constants $a$ and $d$ represent the birth rate and the death rate, respectively. $\alpha_{1}>0$ and $\alpha_{2}>0$ are constants which depend on the specific species. The equation may have one $N=N^{*}$ (or two) positive equilibrium solutions which satisfies $N^{\prime}(t)=0$, and $N=N^{*}$ is usually called the balance state of the system.

In the case of the long range foraging behavior, the species inhabiting two patches may transfer to each other which leads to the change of the model of the 
species growth. Denote the population densities in Patch $A$ and $B$ by $x(t)$ and $y(t)$, respectively, then we propose a model as follows:

$$
\left\{\begin{array}{l}
x^{\prime}(t)=a x(t)\left(1-k_{1} x-k_{2} x^{2}\right)-d x+T_{x}+\sigma(t, x) \dot{\xi}_{t}(\omega), \\
y^{\prime}(t)=a y(t)\left(1-b_{1} y-b_{2} y^{2}\right)-d y+T_{y}
\end{array}\right.
$$

where constants $k_{i}$ and $b_{i}(i=1,2)$ are distinct to indicate the limitation effects of resources, because the abundance degrees of food resources in two patches are often different. $T_{x}$ and $T_{y}$ represent the density change due to transferring. In order to seek for ample food resources, the species inhabiting one patch may transfer to the other by taking some time. Here we assume that the transfer time is a fixed constant $\tau$. The transferring rate is exponentially decaying nonlinear with respect to the time $\tau$ which takes the form $D e^{-p \tau}$, where $p$ represents the death rate during the process of transferring. The population number in each patch varies because of incoming and outgoing population of the species. We assume that in Patch A the incoming number at time $t$ is a definite integral of the density $y(t)$ in the interval of time $\Delta t([t-\Delta t, t])$, and the outgoing number at time $t$ is a definite integral of the density $x(t)$ in the time interval $[t-\tau-\triangle t, t-\tau]$. Hence, when $\triangle t$ is sufficiently small, we obtain the density change in Patch A at time $t$ is $T_{x}=D e^{-p \tau}(y(t)-x(t-\tau))$. Similarly, the density change in Patch B at time $t$ is derived as $T_{y}=D e^{-p \tau}(x(t)-y(t-\tau))$. The term $\xi_{t}(\omega)$ is a stochastic process which represents the noise term. In our study this noise is internal to the system because of random fluctuations in the system parameters. To gain insight into the effect of noise on the system, we assume the noise to be Gaussian distributed white noise with zero mean and a delta function autocorrelation $<\xi_{t} \xi_{s}>=\delta(t-s)$. In our study, we only consider the case where one patch is affected by the noise factor. So far as our best knowledge goes, it seems that the reference for the noise terms on both patches is not available yet.

Note that without noise system (1) is the delay differential model with a nonlinear delay dependant coefficient $D e^{-p \tau}$. Many elegant results have been established in the literature [8-15]. Ailello and his co-workers [8, 9] showed that under appropriate assumptions all solutions are positive and bounded. Criteria for the existence of positive equilibria and the stability of the equilibria are also discussed. Kuang and Beretta etc. [10-12] proposed practically perfect guidelines that combine graphical information with analytical work to effectively study the local stability of some models of Bence and Nisbet involving delay dependent parameters. Specifically, it was shown that the stability of a given steady state is determined by the graphs of some functions of $\tau$ which can be expressed explicitly. In [13-15], it was shown that the delay can qualitatively change the dynamics. For some fixed values of the parameters, as the delay increases the equilibrium can switch from being stable to unstable (with numerically observed periodic solutions) and then back to stable. Lei and Mackey [16] studied the moment stability of the trivial solution of a linear differential delay equation in the presence of multiplicative white noise and applied their results to examining the local stability of the hematopoietic stem cell regulation system in the presence of noise. Lotka-Volterra type predator-prey systems with stage structure and time delays under various assumptions were investigated 
in [17-19]. For the role of noise in delay models and the stochastic differential delay equations, and applications of bifurcations in dynamical systems, we refer the reader to the literature [20-22].

The rest of the paper is organized as follows. In Section 2, we discuss dynamics of system (1) without the noise effect, and aim to develop an effective geometrical method to study the local stability of the balance states. By integrating the graphical information into analytical work, the stability area and Hopf bifurcation curves in the $(\tau-p)$ parameter plane are depicted. In Section 3, the bifurcation direction and the stability of bifurcating periodic solution are investigated. By applying the center manifold theorem, the Poincaré normal form is found and the approximate periodic solution near the critical delay values is obtained. In Section 4, the complete synchronization of variations of the population size of species in two patches is analyzed qualitatively and numerically. The moment stability of the solution of the stochastic delay equation is also studied by applying the Itô integral. Section 5 presents a brief conclusion.

\section{Stability Analysis of the Balance State $\left(x^{*}, y^{*}\right)$ Without the Noise Term}

Suppose that $\left(x^{*}, y^{*}\right)$ is the positive equilibrium solution of system (1) without the noise term. Here, for the convenience of our study, we assume $x^{*}=y^{*}$ because in this case it is translation invariant. Namely, after making the coordinate transformations $\bar{x}=x-x^{*}, \bar{y}=y-y^{*}$ we transform the balance state $\left(x^{*}, y^{*}\right)$ to the origin $(0,0)$. The resultant system preserves the same form as system (1) under certain parametric conditions, and local behaviors at $\left(x^{*}, y^{*}\right)$ of the original system and at $(0,0)$ of the resultant system are topologically equivalent. Therefore, hereafter we only need to focus on the stability of the origin $(0,0)$ of system (1).

Here we suppose $r=d-a>0$, which means the death rate is larger than the birth rate. The linear form of system (1) at the origin is

$$
\left\{\begin{array}{l}
x^{\prime}(t)=-r x(t)+D e^{-p \tau}(y(t)-x(t-\tau)), \\
y^{\prime}(t)=-r y(t)+D e^{-p \tau}(x(t)-y(t-\tau)) .
\end{array}\right.
$$

The corresponding characteristic equation of (2) is given as

$$
\Delta(\lambda, \tau)=\left(r+\lambda+D e^{-(\lambda+p) \tau}\right)^{2}-D^{2} e^{-2 p \tau}=0 .
$$

When $\tau=0$, it becomes

$$
\Delta(\lambda, 0)=(r+\lambda+D)^{2}-D^{2}=0 .
$$

From equation (4), we can see that the trivial solution of system (2) is stable in the case of $\tau=0$.

For the case of $\tau>0$, we suppose that $\lambda= \pm i \beta(\beta>0)$ are two roots of equation (3). Substituting it into equation (3) and equating coefficients of the real part and the imaginary part to zero, respectively, yields

$$
\left\{\begin{array}{l}
D \sin (\beta \tau)=e^{p \tau} \beta, \\
D \cos (\beta \tau)=D-r e^{p \tau} .
\end{array}\right.
$$


Letting $\beta \tau=T$, then from equation (5), we have

$$
\left\{\begin{array}{l}
\tau=\frac{1}{p} \ln \frac{D(1-\cos T)}{r}, \\
p=\frac{r \sin T}{1-\cos T} \frac{1}{T} \ln \frac{D(1-\cos T)}{r} .
\end{array}\right.
$$

When $2 k \pi<T<(2 k+1) \pi(k=0,1,2, \cdots)$, we get that $\sin T>0$ and the boundary condition is given as $D>\frac{r}{2}$. Assume $T_{0}=T-2 k \pi$ for every $k=$ $0,1,2, \cdots$, then $T_{0}$ is limited to the range $0<T_{0}<\pi$. By the second equation of (6), we deduce that, for any fixed values of $D$ and $r, p$ continuously varies in the range $\left[\arccos \left(1-\frac{D}{r}\right)+2 k \pi,(2 k+1) \pi\right]$, and the maximal value $p_{k}^{\max }$ can be attained for each $k$. If we fix the parameters as $r=2.0$ and $D=3.0$, the curves of parameter $p$ versus $T_{0}$ are depicted for the cases of $k=0,1,2$ in Figure 2.

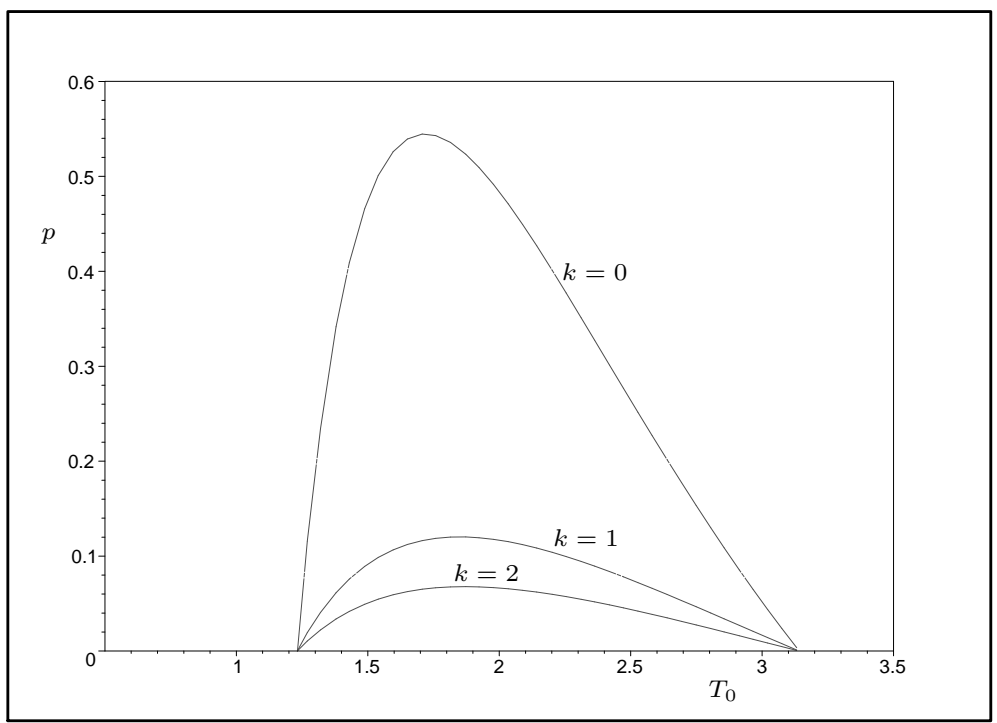

Figure 2. From the top to the bottom, the curves of parameter $p$ versus $T_{0}$ for the cases of $k=0,1,2$, in the $\left(p-T_{0}\right)$ plane with parameters $r=2.0$ and $D=3.0$.

From the above analysis, we can see that if $p>p_{0}^{\max }$, no imaginary roots exist for equation (3), and system (2) is asymptotically stable for every $\tau \geq 0$. If $p_{k}^{\max }<p<p_{k-1}^{\max }$, there are $2 k(k=1,2, \cdots)$ pairs of imaginary roots for equation (3) in total. If $p=p_{k}^{\max }$ for some $k$, there are $2 k+1(k=0,1,2, \cdots)$ pairs of imaginary roots for equation (3). We denote the corresponding delay bifurcation values by $\tau_{1, j}$ and $\tau_{2, j}$ from the left to the right for $j=1,2, \cdots, k$.

Suppose that the characteristic equation (3) has roots $\lambda(\tau)=\alpha(\tau) \pm i \nu(\tau)$, which satisfy $\alpha\left(\tau_{c}\right)=0$ and $\nu\left(\tau_{c}\right)=\beta$ with $\tau_{c}=\tau_{1, k}$ or $\tau_{2, k}(k=1,2, \cdots)$. By 
substituting it into equation (3), we have

$$
\begin{aligned}
\alpha+r+D e^{-(p+\alpha) \tau} \cos (\nu \tau)-D e^{-p \tau} & =0 \\
\nu-D e^{-(p+\alpha) \tau} \sin (\nu \tau) & =0
\end{aligned}
$$

Differentiating both side of equation (7) with respect to $\tau$ gives

$$
\begin{aligned}
& \left(1-D e^{-p \tau} \cos (\nu \tau) \tau\right) \frac{\mathrm{d} \alpha}{\mathrm{d} \tau}-D e^{-p \tau} \sin (\nu \tau) \tau \frac{\mathrm{d} \nu}{\mathrm{d} \tau} \\
& =D e^{-p \tau}[p \cos (\nu \tau)+\sin (\nu \tau) \nu-p], \\
& D \tau e^{-p \tau} \sin (\nu \tau) \frac{\mathrm{d} \alpha}{\mathrm{d} \tau}+\left(1-D e^{-p \tau} \cos (\nu \tau) \tau\right) \frac{\mathrm{d} \nu}{\mathrm{d} \tau} \\
& =-D e^{-p \tau}[p \sin (\nu \tau)-\nu \cos (\nu \tau)] .
\end{aligned}
$$

Solving $\frac{\mathrm{d} \alpha}{\mathrm{d} \tau}$ from equation (8) we have

(9)

$$
\begin{aligned}
\left.\frac{\mathrm{d} \alpha}{\mathrm{d} \tau}\right|_{\tau=\tau_{c}} & =\frac{D e^{p \tau}\left[-p+p \cos (\beta \tau)-D e^{-p \tau} p \tau+\beta \sin (\beta \tau)+D p e^{-p \tau} \cos (\beta \tau) \tau\right]}{e^{2 p \tau}-2 e^{p \tau} D \cos (\beta \tau) \tau+D^{2} \tau^{2}} \\
& =\frac{r^{2}(\cos T+1)\left(T(1+\cos T)-(\sin T+T) \ln \frac{-D(\cos T-1)}{r}\right)}{T\left(1+T^{2}-2 T \cos T \sin T-\cos ^{2} T\right)}
\end{aligned}
$$

Using a similar argument as the above, we can obtain the maximal value $p_{k}^{\max }$ for $p$ by differentiating both sides of the second equation of (6) with respect to $T$

$$
\frac{d p}{d T}=\frac{r\left(T(1+\cos T)-(T+\sin T) \ln \frac{D(1-\cos T)}{r}\right)}{T^{2}(1-\cos T)} .
$$

When $p$ attains the maximal value $p_{k}^{\max }$, we have $\frac{d p}{d T}=0$. Denote the corresponding maximal values of $T$ and $\tau$ by $T_{k}^{\max }$ and $\tau_{k}^{\max }$, respectively, then from (10) we get

for $k=0,1,2, \cdots$.

$$
\frac{\mathrm{d} p}{\mathrm{~d} T}\left\{\begin{array}{cc}
>0, & \text { if } \quad T<T_{\max }^{k}, \\
<0, & \text { if } \quad T>T_{\max }^{k},
\end{array}\right.
$$

Combining equations (7) and (9), we have

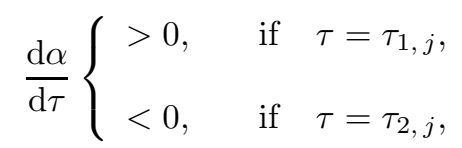

for $j=0,1, \cdots, k$. For the fixed parameters $r=2$ and $D=3.0$, in Figure 3 it shows the Hopf bifurcation curves of system $(2)$ in the $(p, \tau)$ plane for $k=0,1,2$. The shaded part represents the stable region.

We can see from Figure 3 if the value of $p$ is fixed, the trivial solution of system (2) experiences three stability switches from being stable to unstable, and then back to stable. Inside the region enclosed by the Hopf bifurcation curves, the complex dynamical behavior may occur. 


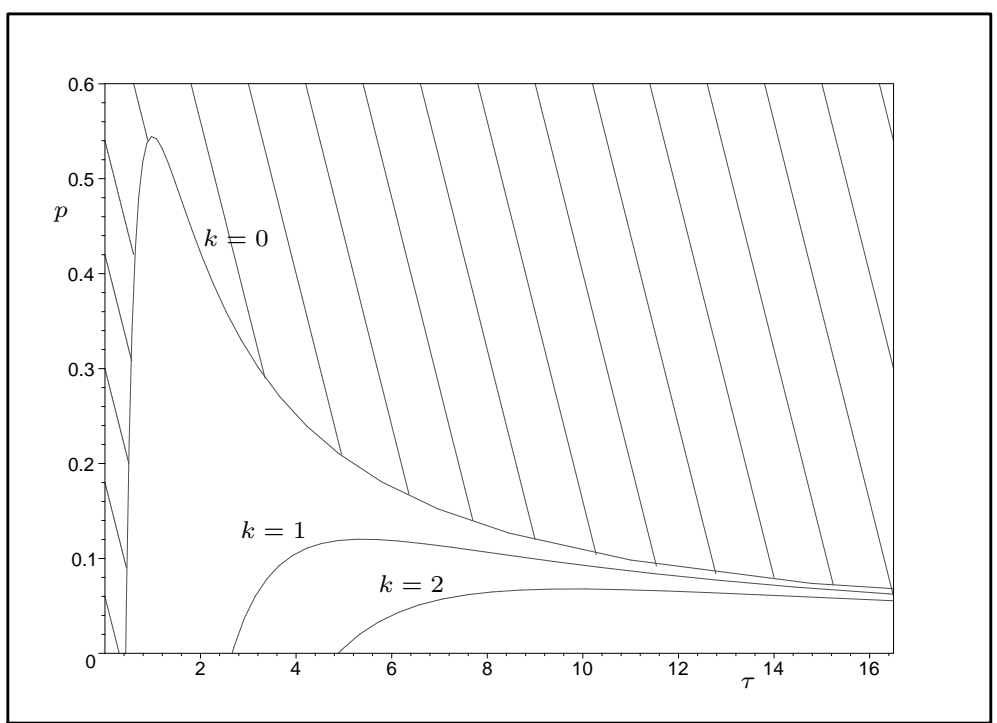

FiguRE 3. From the top to the bottom, the Hopf bifurcation curves of system (2) for the cases of $k=0,1,2$, in the $(p-\tau)$ plane with parameters $r=2.0$ and $D=3.0$. The shaded part represents the stable regime.

\section{Poincaré Normal Form}

In this section, the bifurcation direction and the stability of bifurcating periodic solutions, in a simple Hopf bifurcation of system (1) without noise at $\tau=\tau_{c}$ presented in the preceding section, are considered by means of the center manifold theory and the Poincaré normal form technique.

Set $\sigma(t, x)=0$. System (1) can be rewritten as

$$
\left[\begin{array}{l}
x^{\prime}(t) \\
y^{\prime}(t)
\end{array}\right]=M\left[\begin{array}{l}
x(t) \\
y(t)
\end{array}\right]+N\left[\begin{array}{l}
x(t-\tau) \\
y(t-\tau)
\end{array}\right]+\left[\begin{array}{c}
-s_{1} x^{2}(t)-s_{2} x^{3}(t) \\
-l_{1} y^{2}(t)-l_{2} y^{3}(t)
\end{array}\right]
$$

with

$$
M=\left[\begin{array}{cc}
-r & D e^{-p \tau} \\
D e^{-p \tau} & -r
\end{array}\right], \quad N=\left[\begin{array}{cc}
-D e^{-p \tau} & 0 \\
0 & -D e^{-p \tau}
\end{array}\right] .
$$

Choose the phase space as $\mathbb{C}=\mathbb{C}\left([-\tau, 0], \mathbb{R}^{2}\right)$. For $\phi=\left(\phi_{1}(\theta), \phi_{2}(\theta)\right)^{T} \in \mathbb{C}$, we define

$$
L \phi=\int_{-\tau}^{0}[\mathrm{~d} \eta(\theta)] \phi(\theta)
$$

where $\eta:[-\tau, 0] \rightarrow \mathbb{R}^{2} \times \mathbb{R}^{2}$ is a $2 \times 2$ bounded variation function with

$$
\mathrm{d} \eta(\theta)=\left[\begin{array}{cc}
-r \delta(\theta)-D e^{-p \tau} \delta(\theta+\tau) & D e^{-p \tau} \delta(\theta) \\
D e^{-p \tau} \delta(\theta) & -r \delta(\theta)-D e^{-p \tau} \delta(\theta+\tau)
\end{array}\right] \mathrm{d} \theta .
$$


For any $\phi \in \mathbb{C}$, the linear operator defined by (13) generates a strong continuous semigroup of bounded linear operators with the infinitesimal generator

$$
\mathcal{A} \phi= \begin{cases}\frac{\mathrm{d} \phi}{\mathrm{d} \theta}, & \theta \in[-\tau, 0) \\ L \phi, & \theta=0\end{cases}
$$

and

$$
\mathcal{Q}(\phi)=\left\{\begin{aligned}
0, & \theta \in[-\tau, 0), \\
F(\phi), & \theta=0
\end{aligned}\right.
$$

where

$$
F(\phi)=\left(\begin{array}{c}
-s_{1} \phi_{1}^{2}(0)-s_{2} \phi_{1}^{3}(0) \\
-l_{1} \phi_{2}^{2}(0)-l_{2} \phi_{2}^{3}(0)
\end{array}\right) .
$$

As for the parametric case of $\tau=\tau_{c}$, system (11) can be written as a operator differential equation

$$
u^{\prime}(t)=\mathcal{A} u_{t}+\mathcal{Q} u_{t},
$$

where $u=(x, y)^{T}$ and $u_{t}=u(t+\theta)$ for $-\tau \leq \theta<0$. Let $\psi \in \mathbb{C}^{*}=\mathbb{C}\left([0, \tau], \mathbb{R}^{2}\right)$ (the dual space of $\mathbb{C}$ ). We define the adjoint operator $\mathcal{A}^{*}$ of $\mathcal{A}$ as

$$
\mathcal{A}^{*} \psi(s)=\left\{\begin{array}{r}
-\frac{\mathrm{d} \psi}{\mathrm{d} s}, \quad 0<s \leq \tau, \\
\int_{-\tau}^{0} \mathrm{~d} \eta^{T}(s) \psi(-s), \quad s=0 .
\end{array}\right.
$$

Thus, for $\varphi \in \mathbb{C}$ and $\psi \in \mathbb{C}^{*}$, we can define a bilinear form:

$$
<\psi, \varphi>=\bar{\psi}^{T}(0) \varphi(0)-\int_{-\tau}^{0} \int_{0}^{\theta} \bar{\psi}^{T}(\xi-\theta) \mathrm{d} \eta(\theta) \varphi(\xi) d \xi .
$$

From the discussions presented in the last section, we know that, when $p<$ $p_{0}^{\max }$ the characteristic equation (3) has a pair of pure imaginary eigenvalues $\Lambda=$ $\{i \beta,-i \beta\}$ at $\tau_{c}=\tau_{1,1}$ and $\tau_{2,1}$, and other eigenvalues with negative real parts. Hence, $\mathbb{C}$ can be decomposed as $\mathbb{C}=P_{\Lambda} \oplus Q_{\Lambda}$, where $P_{\Lambda}$ is the characteristic space corresponding to eigenvalues $\Lambda$, and $Q_{\Lambda}$ is the complementary subspace of $P_{\Lambda}$. We suppose that $q_{1}, q_{2} \in \mathbb{C}$ are two real eigenvectors of the linear operator $\mathcal{A}$ associated with the critical eigenvalue $i \beta$, which satisfy

$$
\mathcal{A} q_{1}(\theta)=-\beta q_{2}(\theta), \quad \mathcal{A} q_{2}(\theta)=\beta q_{1}(\theta) .
$$

Making use of the definition (14), system (17) generates a 4-dimensional coupled linear first-order boundary value problem and its solution is given by

$$
q_{1}(\theta)=\left[\begin{array}{c}
\cos (\beta \theta) \\
\cos (\beta \theta)
\end{array}\right], \quad q_{2}(\theta)=\left[\begin{array}{c}
\sin (\beta \theta) \\
\sin (\beta \theta)
\end{array}\right] .
$$

Eigenvectors $p_{1}, p_{2} \in \mathbb{C}^{*}$ of $\mathcal{A}^{*}$ associated with the eigenvalue $-i \beta$ are determined by a similar boundary value problem

$$
\mathcal{A}^{*} p_{1}(s)=\beta p_{2}(s), \quad \mathcal{A}^{*} p_{2}(s)=-\beta p_{1}(s) .
$$

The use of definition of $\mathcal{A}^{*}$ leads to

$$
p_{1}(s)=\left[\begin{array}{c}
n_{1} \cos (\beta s)+n_{2} \sin (\beta s) \\
n_{1} \cos (\beta s)+n_{2} \sin (\beta s)
\end{array}\right], \quad p_{2}(s)=\left[\begin{array}{c}
-n_{2} \cos (\beta s)+n_{1} \sin (\beta s) \\
-n_{2} \cos (\beta s)+n_{1} \sin (\beta s)
\end{array}\right] .
$$

Thus, the orthogonality conditions

$$
<p_{1}, q_{1}>=1, \quad<p_{1}, q_{2}>=0,
$$


become equivalent to

$$
\left[\begin{array}{cc}
G & -\tau \sin (\beta \tau) D e^{-p \tau} \\
\tau \sin (\beta \tau) D e^{-p \tau} & G
\end{array}\right]\left[\begin{array}{l}
n_{1} \\
n_{2}
\end{array}\right]=\left[\begin{array}{l}
1 \\
0
\end{array}\right]
$$

where

$$
G=-D \tau e^{-p \tau} \cos (\beta \tau)+\frac{D \sin (\beta \tau) e^{-p \tau}}{\beta} .
$$

Solving for $n_{1}$ and $n_{2}$ from equation (19), we have

$$
\begin{aligned}
& n_{1}=\frac{\beta(-\cos (\beta \tau) \tau \beta+\sin (\beta \tau))}{D e^{-p \tau}\left(\tau^{2} \beta^{2}-2 \cos (\beta \tau) \tau \beta \sin (\beta \tau)+\sin ^{2}(\beta \tau)\right)}, \\
& n_{2}=\frac{-\tau \sin (\beta \tau) \beta^{2}}{D e^{-p \tau}\left(\tau^{2} \beta^{2}-2 \cos (\beta \tau) \tau \beta \sin (\beta \tau)+\sin ^{2}(\beta \tau)\right)} .
\end{aligned}
$$

Set

$$
\begin{array}{ll}
\Phi(\theta)=\left(q_{1}(\theta), q_{2}(\theta)\right), & -\tau \leq \theta \leq 0, \\
\Psi(s)=\left(p_{1}(s), p_{2}(s)\right), & 0 \leq s \leq \tau .
\end{array}
$$

We re-express $u_{t} \in \mathbb{C}$ as

$$
u_{t}=\Phi z+w
$$

with $z=\left(z_{1}, z_{2}\right)^{T}$, and $\Phi z$ is the projection vector of $u_{t}$ onto the center manifold. Since

$$
<\Psi, \Phi z^{\prime}+w^{\prime}>=<\Psi,(\mathcal{A}+\mathcal{Q})(\Phi z+w)>
$$

using the definition of innerproduct (16) and the definition of operator $\mathcal{Q}$ as given in equation (15), from (21) we obtain a resultant ODE system as $(22)$

$$
\begin{aligned}
{\left[\begin{array}{l}
z_{1}^{\prime} \\
z_{2}^{\prime} \\
w^{\prime}
\end{array}\right]=} & {\left[\begin{array}{ccc}
0 & \beta & 0 \\
-\beta & 0 & 0 \\
0 & 0 & \mathcal{A}
\end{array}\right]\left[\begin{array}{c}
z_{1} \\
z_{2} \\
w
\end{array}\right] } \\
& +\left[\begin{array}{l}
p_{1}^{T}(0) \mathcal{Q}\left(z_{1} q_{1}+z_{2} q_{2}+w\right)(0) \\
p_{2}^{T}(0) \mathcal{Q}\left(z_{1} q_{1}+z_{2} q_{2}+w\right)(0) \\
-\sum_{j=1,2} p_{j}^{T}(0) \mathcal{Q}\left(z_{1} q_{1}+z_{2} q_{2}+w\right)(0) q_{j}+\mathcal{Q}\left(z_{1} q_{1}+z_{2} q_{2}+w\right)
\end{array}\right]
\end{aligned}
$$

With the aid of formulas (14)-(18), the right hand side of system (22) can be decomposed in the form:

$$
\begin{aligned}
& {\left[\begin{array}{l}
z_{1}^{\prime} \\
z_{2}^{\prime} \\
w^{\prime}
\end{array}\right]=\left[\begin{array}{ccc}
0 & \beta & 0 \\
-\beta & 0 & 0 \\
0 & 0 & \mathcal{A}
\end{array}\right]\left[\begin{array}{l}
z_{1} \\
z_{2} \\
w
\end{array}\right]+\left[\begin{array}{l}
-n_{1}\left(l_{1}+s_{1}\right) z_{1}^{2}-n_{1}\left(l_{2}+s_{2}\right) z_{1}^{3} \\
n_{2}\left(l_{1}+s_{1}\right) z_{1}^{2}+n_{2}\left(l_{2}+s_{2}\right) z_{1}^{3} \\
H
\end{array}\right]} \\
& +\left[\begin{array}{ll}
-2 n_{1} z_{1}\left(l_{1} w_{2}+s_{1} w_{1}\right)(0) & \\
2 n_{2} z_{1}\left(l_{1} w_{2}+s_{1} w_{1}\right)(0) & \text { if } \quad-\tau \leq \theta<0 \\
0 & \text { if } \theta=0 \\
{\left[\begin{array}{l}
-s_{1} z_{1}^{2} \\
-l_{1} z_{1}^{2}
\end{array}\right]} &
\end{array}\right],
\end{aligned}
$$

where

$$
H=\left(l_{1}+s_{1}\right) z_{1}^{2}\left[\begin{array}{l}
1 \\
1
\end{array}\right]\left(n_{1} \cos (\beta \theta)-n_{2} \sin (\beta \theta)\right) .
$$

The plane spanned by eigenvectors $q_{1}$ and $q_{2}$ is tangent to the center manifold at the origin. This implies that by using the Taylor Series the center manifold can 
be approximated locally as a truncated power series of $w$ depending on the second power of the coordinates $z_{1}$ and $z_{2}$ as

$$
w(\theta)=\frac{1}{2}\left(h_{20}(\theta) z_{1}^{2}+2 h_{11}(\theta) z_{1} z_{2}+h_{02}(\theta) z_{2}^{2}\right) .
$$

So the derivative of $w$ with respect to $\theta$ is

$$
w^{\prime}(\theta)=-\beta h_{11}(\theta) z_{1}^{2}+\beta\left(h_{20}(\theta)-h_{02}(\theta)\right) z_{1} z_{2}+\beta h_{11}(\theta) z_{2}^{2} .
$$

Note that the formula of the derivative $w^{\prime}(\theta)$ can also be expressed by the third equation of system (23). The comparison of the coefficients of $z_{1}^{2}, z_{1} z_{2}$ and $z_{2}^{2}$ yields a linear boundary value problem for the unknown coefficients of the center manifold, where the resultant differential system is

$$
\left\{\begin{array}{l}
h_{20}^{\prime}(\theta)=-2 \beta h_{11}(\theta)-2\left(l_{1}+s_{1}\right)\left[\begin{array}{l}
1 \\
1
\end{array}\right]\left(n_{1} \cos (\beta \theta)-n_{2} \sin (\beta \theta)\right), \\
h_{11}^{\prime}(\theta)=\beta h_{20}(\theta)-\beta h_{02}(\theta) \\
h_{02}^{\prime}(\theta)=2 \beta h_{11}(\theta)
\end{array}\right.
$$

with boundary conditions

$$
\left\{\begin{array}{l}
M h_{20}(0)+N h_{20}(-\tau)=-2 \beta h_{11}(0)-2 n_{1}\left(l_{1}+s_{1}\right)\left[\begin{array}{l}
1 \\
1
\end{array}\right]+2\left[\begin{array}{l}
s_{1} \\
l_{1}
\end{array}\right], \\
M h_{11}(0)+N h_{11}(-\tau)=\beta h_{20}(0)-\beta h_{02}(0), \\
M h_{02}(0)+N h_{02}(-\tau)=2 \beta h_{11}(0),
\end{array}\right.
$$

where $n_{1}$ and $n_{2}$ are the same as (20), and the definition of matrix $M$ and $N$ are given as in (12). Solving system (25) we obtain

$$
\left\{\begin{aligned}
h_{20}(\theta)= & \frac{-2\left(l_{1}+s_{1}\right)\left(n_{2} \cos (\beta \theta)+n_{1} \sin (\beta \theta)\right)}{3 \beta} \mathrm{I} \\
& +E_{1}-E_{2} \sin (2 \beta \theta)+E_{3} \cos (2 \beta \theta), \\
h_{11}(\theta)= & \frac{-2\left(l_{1}+s_{1}\right)\left(n_{1} \cos (\beta \theta)-n_{2} \sin (\beta \theta)\right)}{3 \beta} \mathrm{I} \\
& +E_{2} \cos (2 \beta \theta)+E_{3} \sin (2 \beta \theta), \\
h_{02}(\theta)= & \frac{-4\left(l_{1}+s_{1}\right)\left(n_{2} \cos (\beta \theta)+n_{1} \sin (\beta \theta)\right)}{3 \beta} \mathrm{I} \\
& +E_{1}+E_{2} \sin (2 \beta \theta)-E_{3} \cos (2 \beta \theta),
\end{aligned}\right.
$$

where $\mathrm{I}$ is the identity matrix and $E_{i j}$ are determined by boundary conditions (26) as

$$
E_{1}=\left[\begin{array}{c}
E_{11} \\
E_{12}
\end{array}\right], \quad E_{2}=\left[\begin{array}{c}
E_{21} \\
E_{22}
\end{array}\right], \quad E_{3}=\left[\begin{array}{c}
E_{31} \\
E_{32}
\end{array}\right],
$$

where

$$
\begin{gathered}
E_{11}=-\frac{s_{1} r e^{p \tau}+D\left(l_{1}+s_{1}\right)}{r\left(r e^{p \tau}+2 D\right)}, \\
E_{12}=-\frac{l_{1} r e^{p \tau}+D\left(l_{1}+s_{1}\right)}{r\left(r e^{p \tau}+2 D\right)}, \\
E_{21}=\frac{-2 D \sin (\beta \tau)\left[2 D^{2}\left(3 r e^{p \tau}-D\right)\left(s_{1}+l_{1}\right)+\left(4 r s_{1} e^{p \tau}-4 l_{1} D-13 s_{1} D\right) r^{2} e^{2 p \tau}\right]}{r\left(36 D^{4}+16 r^{4} e^{4 p \tau}-104 r^{3} D e^{3 p \tau}-124 r D^{3} e^{p \tau}+201 r^{2} D^{2} e^{2 p \tau}\right)}, \\
E_{22}=\frac{-2 D \sin (\beta \tau)\left[2 D^{2}\left(3 r e^{p \tau}-D\right)\left(s_{1}+l_{1}\right)+\left(4 r l_{1} e^{p \tau}-4 s_{1} D-13 l_{1} D\right) r^{2} e^{2 p \tau}\right]}{r\left(36 D^{4}+16 r^{4} e^{4 p \tau}-104 r^{3} D e^{3 p \tau}-124 r D^{3} e^{p \tau}+201 r^{2} D^{2} e^{2 p \tau}\right)},
\end{gathered}
$$




$$
\begin{aligned}
E_{31} & =\frac{6 D^{4}\left(l_{1}+s_{1}\right)+D r l_{1} e^{p \tau}\left(20 D r e^{p \tau}-13 D^{2}-8 e^{2 p \tau} r^{2}\right)}{r\left(36 D^{4}+16 r^{4} e^{4 p \tau}-104 r^{3} D e^{3 p \tau}-124 r D^{3} e^{p \tau}+201 r^{2} D^{2} e^{2 p \tau}\right)} \\
& +\frac{r s_{1} e^{p \tau}\left(55 D^{2} r e^{p \tau}+8 e^{3 p \tau} r^{3}-38 e^{2 p \tau} r^{2} D-31 D^{3}\right)}{r\left(36 D^{4}+16 r^{4} e^{4 p \tau}-104 r^{3} D e^{3 p \tau}-124 r D^{3} e^{p \tau}+201 r^{2} D^{2} e^{2 p \tau}\right)}, \\
E_{32} & =\frac{6 D^{4}\left(l_{1}+s_{1}\right)+D r s_{1} e^{p \tau}\left(20 D r e^{p \tau}-13 D^{2}-8 e^{2 p \tau} r^{2}\right)}{r\left(36 D^{4}+16 r^{4} e^{4 p \tau}-104 r^{3} D e^{3 p \tau}-124 r D^{3} e^{p \tau}+201 r^{2} D^{2} e^{2 p \tau}\right)}, \\
& +\frac{r l_{1} e^{p \tau}\left(55 D^{2} r e^{p \tau}+8 e^{3 p \tau} r^{3}-38 e^{2 p \tau} r^{2} D-31 D^{3}\right)}{r\left(36 D^{4}+16 r^{4} e^{4 p \tau}-104 r^{3} D e^{3 p \tau}-124 r D^{3} e^{p \tau}+201 r^{2} D^{2} e^{2 p \tau}\right)}
\end{aligned}
$$

With formulas (27) and system (24), the approximate equation of the center manifold can be reconstructed. Substituting $w_{1}$ and $w_{2}$ into the equation of $w(\theta)$, we obtain the following system that describes the flow restricted to the 2-dimensional center manifold:

$$
\left[\begin{array}{l}
z_{1}^{\prime} \\
z_{2}^{\prime}
\end{array}\right]=\left[\begin{array}{cc}
0 & \beta \\
-\beta & 0
\end{array}\right]\left[\begin{array}{l}
z_{1} \\
z_{2}
\end{array}\right]+\left[\begin{array}{l}
\sum_{j, k>0}^{j+k=2,3} g_{j k}^{(1)} z_{1}^{j} z_{2}^{k} \\
\sum_{j, k \geq 0}^{j+k=2,3} g_{j k}^{(2)} z_{1}^{j} z_{2}^{k}
\end{array}\right]
$$

where

$$
\begin{aligned}
g_{20}^{(1)}= & -n_{1}\left(l_{1}+s_{1}\right), \quad g_{11}^{(1)}=g_{02}^{(1)}=g_{03}^{(1)}=0 \\
g_{30}^{(1)}= & -2 n_{1}\left[l_{1}\left(\frac{1}{2} E_{32}-\frac{1}{3 \beta} n_{2} l_{1}-\frac{1}{3 \beta} n_{2} s_{1}+\frac{1}{2} E_{12}\right)+\right. \\
& \left.s_{1}\left(\frac{1}{2} E_{31}-\frac{1}{3 \beta} n_{2} l_{1}-\frac{1}{3 \beta} n_{2} s_{1}+\frac{1}{2} E_{11}\right)\right]-n_{1}\left(l_{2}+s_{2}\right), \\
g_{21}^{(1)}= & -2 n_{1}\left[l_{1}\left(E_{22}-\frac{2}{3 \beta} n_{1} s_{1}-\frac{2}{3 \beta} n_{1} l_{1}\right)+s_{1}\left(E_{21}-\frac{2}{3 \beta} n_{1} s_{1}-\frac{2}{3 \beta} n_{1} l_{1}\right)\right], \\
g_{12}^{(1)}=- & -2 n_{1}\left[\frac{1}{2} l_{1}\left(-E_{32}-\frac{4}{3 \beta} n_{2} l_{1}-\frac{4}{3 \beta} n_{2} s_{1}+E_{12}\right)\right. \\
+ & \left.\frac{1}{2} s_{1}\left(-E_{31}-\frac{4}{3 \beta} n_{2} l_{1}-\frac{4}{3 \beta} n_{2} s_{1}+E_{11}\right)\right], \\
g_{20}^{(2)}= & n_{2}\left(l_{1}+s_{1}\right), \quad g_{11}^{(2)}=g_{02}^{(2)}=g_{03}^{(2)}=0, \\
g_{30}^{(2)}= & 2 n_{2}\left[l _ { 1 } \left(\frac{1}{2} E_{32}-\frac{1}{3 \beta} n_{2} l_{1}-\frac{1}{3 \beta} n_{2} s_{1}+\right.\right. \\
& \left.\left.\frac{1}{2} E_{12}\right)+s_{1}\left(\frac{1}{2} E_{31}-\frac{1}{3 \beta} n_{2} l_{1}-\frac{1}{3 \beta} n_{2} s_{1}+\frac{1}{2} E_{11}\right)\right]+n_{2}\left(l_{2}+s_{2}\right),
\end{aligned}
$$




$$
\begin{aligned}
g_{21}^{(2)}= & 2 n_{2}\left[l_{1}\left(E_{22}-\frac{2}{3 \beta} n_{1} s_{1}-\frac{2}{3 \beta} n_{1} l_{1}\right)+s_{1}\left(E_{21}-\frac{2}{3 \beta} n_{1} s_{1}-\frac{2}{3 \beta} n_{1} l_{1}\right)\right], \\
g_{12}^{(2)}= & 2 n_{2}\left[\frac{1}{2} l_{1}\left(-E_{32}-\frac{4}{3 \beta} n_{2} l_{1}-\frac{4}{3 \beta} n_{2} s_{1}+E_{12}\right)\right. \\
& \left.+\frac{1}{2} s_{1}\left(-E_{31}-\frac{4}{3 \beta} n_{2} l_{1}-\frac{4}{3 \beta} n_{2} s_{1}+E_{11}\right)\right] .
\end{aligned}
$$

By using the Bautin formula [2], the Poincaré-Lyapunov constant can be found in the Poincaré normal form of system (28) as

$$
\triangle=\frac{1}{8}\left(\frac{2}{\beta} g_{20}^{(1)} g_{20}^{(2)}+g_{30}^{(1)}+g_{12}^{(1)}+g_{21}^{(2)}\right) .
$$

So the bifurcation is supercritical if $\Delta<0$ or subcritical if $\Delta>0$. The amplitude of the stable (unstable) oscillation is given by

$$
A=\sqrt{-\frac{1}{\triangle} \operatorname{Re} \frac{\mathrm{d} \lambda_{1,2}\left(\tau_{c}\right)}{\mathrm{d} \tau}\left(\tau-\tau_{c}\right)}
$$

When $\tau$ is not close to the critical value $\tau_{c}$, the approximation periodic solution is obtained as

$$
\begin{aligned}
& x(t) \approx A \cos (\beta t) \\
& y(t) \approx A \cos (\beta t)
\end{aligned}
$$

where $\beta$ is the vibrational frequency corresponding to the critical point $\tau=\tau_{c}$.

\section{Moment Stability with the Noise Perturbation}

Noise is internal to system (1) since both the birth and the death are randomly varying due to the outer environment, season change, climate and weather, etc. For simplicity, we assume that the function $\sigma$ does not depend on $t$ explicitly. Using the definition of the Gaussian white noise $\xi_{t}$ as the derivative of the Wiener process $W(t)$, we can rewrite system (1) as

$$
\left\{\begin{array}{l}
d x=-r x d t-s_{1} x^{2} d t-s_{2} x^{3} d t+D e^{-p \tau}(y(t)-x(t-\tau)) d t+\sigma(x) \cdot d W(t) \\
d y=-r y d t-l_{1} y^{2} d t-l_{2} y^{3} d t+D e^{-p \tau}(x(t)-y(t-\tau)) d t .
\end{array}\right.
$$

Set $u(t)=(x(t), y(t))^{T}$, then the linear stochastic differential equation of (30) can be re-expressed as

$$
d u(t)=M u(t) d t+N u(t-\tau) d t+\left(\Theta_{0} u(t)+\Theta_{1}\right) d W(t),
$$

where the matrices $M$ and $N$ are given as in (12), and $\Theta_{0}$ and $\Theta_{1}$ are

$$
\Theta_{0}=\left(\begin{array}{cc}
\sigma_{0} & 0 \\
0 & 0
\end{array}\right), \quad \Theta_{1}=\left(\begin{array}{c}
\sigma_{1} \\
0
\end{array}\right) .
$$

Let $U(t)$ be the fundamental solution of equation (31) for $t \geq 0$ and satisfy the initial condition

$$
U(t)= \begin{cases}O, & t<0 \\ I, & t=0\end{cases}
$$

where $O$ is the zero solution and $I$ is the identity matrix of $2 \times 2$. Suppose that $\varphi(\theta) \in \mathbb{C}\left([-\tau, 0], \mathbb{R}^{2}\right)$ with the norm definition $\|\varphi\|=\sup _{-\tau \leq \theta \leq 0}\left|\varphi(\theta)^{T} \xi\right|$, for 
any vector $\xi$ with the character $\|\xi\|=1$. If $\sigma_{0}=\sigma_{1}=0$, using the fundamental solution matrix $U(t)$, the solution of system (31) with the initial condition

$$
\varphi(\theta)=\left(\varphi_{1}(\theta), \varphi_{2}(\theta)\right)^{T} \in \mathbb{C}\left([-\tau, 0], \mathbb{R}^{2}\right),
$$

is given by

$$
u_{\varphi}(t)=U(t) \varphi(0)+\int_{-\tau}^{0} U(t-\tau-s) \varphi(s) d s
$$

If $\sigma_{0}$ or $\sigma_{1}$ is not equal to zero, the solution of equation (32) is a stochastic process given by

$$
u(t ; \varphi)=u_{\varphi}(t)+\int_{0}^{t} U(t-s)\left(\Theta_{0} u(s ; \varphi)+\Theta_{1}\right) d W(s) .
$$

Taking the mathematical expectation of both sides of equation (33), with the Itô interpretation $[16,20]$, we have

$$
E u(t ; \varphi)=E u_{\varphi}(t)=u_{\varphi}(t)
$$

Therefore, the stability condition of the first moment for the solution $u(t, \varphi)$ is identical to that of the unperturbed system and is determined exclusively by $p$ and $\tau$. As discussed in the preceding section, the solution $u_{\varphi}(t)$ of system $(2)$ is asymptotically stable if $(p, \tau)$ lies in the shaded region of Figure 3, which leads to the first moment stability of the solution $u(t, \varphi)$ due to formula (34). Furthermore, since there exists an $\alpha>0$, such that $\left|u_{\varphi}(t)\right| \leq e^{-\alpha t}$ is satisfied, the solution of the nonlinear stochastic delay differential equation (30) has the first moment stability too.

Next, some numerical simulations are undertaken for equation (30) both for the case of noise disappearing and the case of noise arising. We assume $\sigma_{0}=\sigma_{1}=0$, and choose $r=2.0, D=3.0, s_{1}=l_{1}=0, s_{2}=0.2, l_{2}=0.01$ and $p=0.08$, the analysis in Section 2 shows the delay of the Hopf bifurcation to be $\tau_{c}=\{0.4569,13.5884\}$. Using $\tau_{c}$ to compute the bifurcation direction determined by $\triangle$ in equation (29), we obtain $\triangle=-.8050446328 \times 10^{-2}$ and $-.1746873495 \times 10^{-2}$, respectively. That is, the stable periodic solution will appear inside the interval [0.4569, 13.5884]. Choosing $x(t)=0.01$ and $y(t)=0.01$ as $t \in[-\tau, 0]$ and applying the Runge-Kutta method, we obtain the time course and the corresponding phase portraits of three stable periodic solutions for $\tau=0.48,2.98$ and 12.48 as illustrated in Figure 4 (a), (b) and (c), respectively. As shown in Figure 4 (a) and (c), the state variables $x$ and $y$ are almost completely synchronizing when the delay parameter $\tau$ gets close to the Hopf bifurcation value. They change to non-synchronize periodic states when the delay gets far away from the critical value, as shown in Figure 4 (b). To verify the moment stability solution, we take $\sigma_{0}=0.05$ and $\sigma_{1}=0.3$. To be assured $(p, \tau)$ to lie in the shaded region of Figure 3, the delay values are chosen to be $\tau=0.2,0.38$ and $\tau=13.78$, respectively. It is known that the solution should asymptotically tend to zero without the noise effect. However, with choices $\sigma_{0}=0.05$ and $\sigma_{1}=0.3$ we observe oscillatory solutions if noise appears. The expectation values of $x(t, \varphi)$ asymptotically approach to zero, which implies that the solution is the first moment stable since $\operatorname{Ex}(t, \varphi)=x_{\varphi}(t) \rightarrow 0$. 


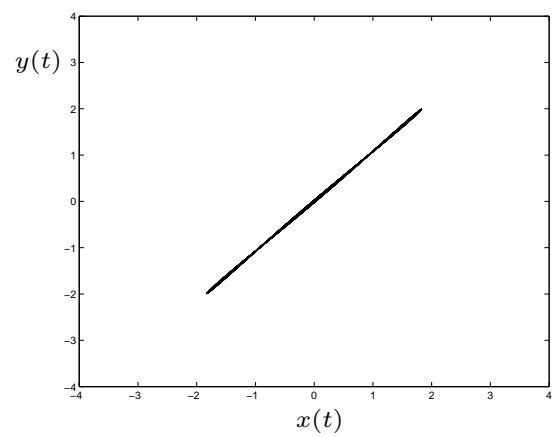

(a)

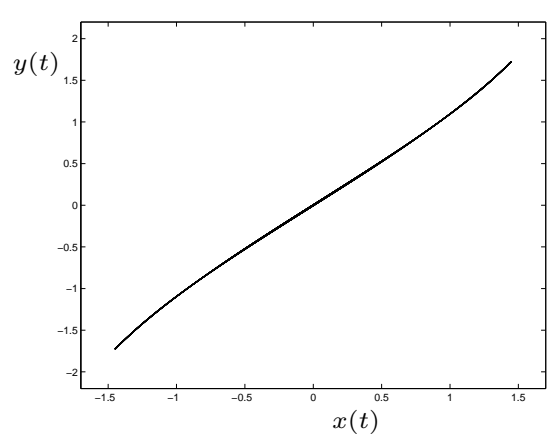

(c)

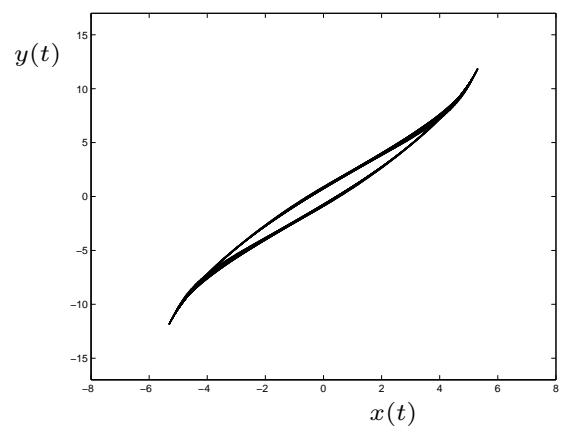

(b)

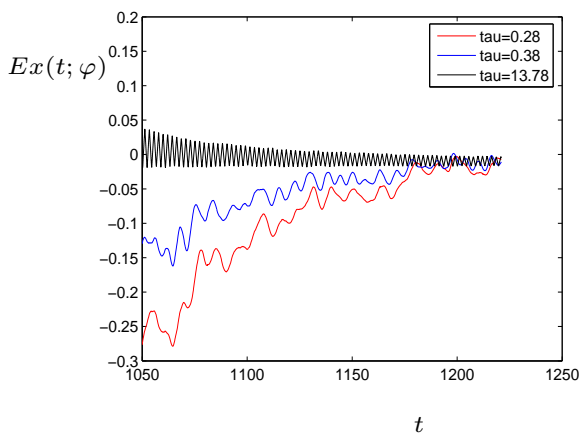

(d)

Figure 4. Phase portraits of system (30) with or without noise. (a) The synchronization phase portrait of $y(t)$ versus $x(t)$ at $\tau=0.48$ when $\sigma_{0}=\sigma_{1}=0$; (b) The non-synchronization phase portrait of $y(t)$ versus $x(t)$ at $\tau=2.98$ when $\sigma_{0}=\sigma_{1}=0$; (c) The phase portrait of $y(t)$ versus $x(t)$ at $\tau=12.48$ when $\sigma_{0}=\sigma_{1}=0$; (d) The expectation values of the stochastic process $x(t ; \varphi)$ asymptotically tend to zero as $t$ gets larger and larger with $\sigma_{0}=0.05$ and $\sigma_{1}=0.3$, where $\tau=0.2,0.38$ and $\tau=13.78$, respectively.

Set $\mathcal{F}_{k}$ to be composed of time series $t_{i, k}$ which satisfies $x(t, \varphi)=0$ and $y(t, \varphi)=0$ at $t=t_{i, k}$ with $k=1,2$, that is

$\mathcal{F}_{1}=\left\{t_{i, 1} \mid x(t, \varphi)=0, d x>0\right.$ at $\left.t=t_{i, 1}\right\}, \mathcal{F}_{2}=\left\{t_{i, 2} \mid y(t, \varphi)=0\right.$ at $\left.t=t_{i, 2}\right\}$.

$(i=1,2, \cdots)$

Furthermore, we define the phase as

$$
\Phi_{k}(t)=\left\{\begin{array}{ll}
\pi \frac{t-t_{i, k}}{t_{i+1, k}-t_{i, k}}, \quad \text { if } \quad t_{i, k} \leq t \leq t_{i+1, k}, & \\
\pi+\pi \frac{t-t_{i+1, k}}{t_{i+2, k}-t_{i+1, k}}, & \text { if } \quad t_{i+1, k} \leq t \leq t_{i+2, k},
\end{array} t_{i, k} \in \mathcal{F}_{k} .\right.
$$




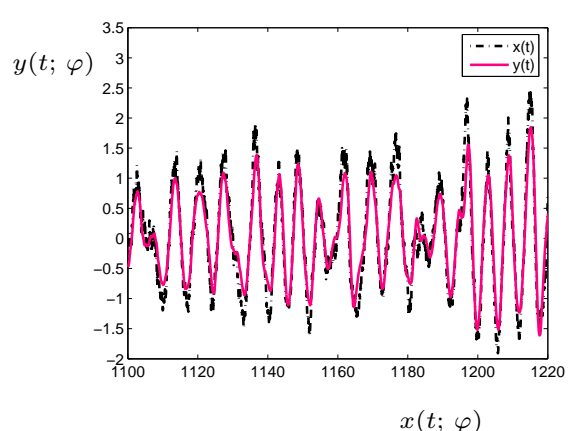

(a)

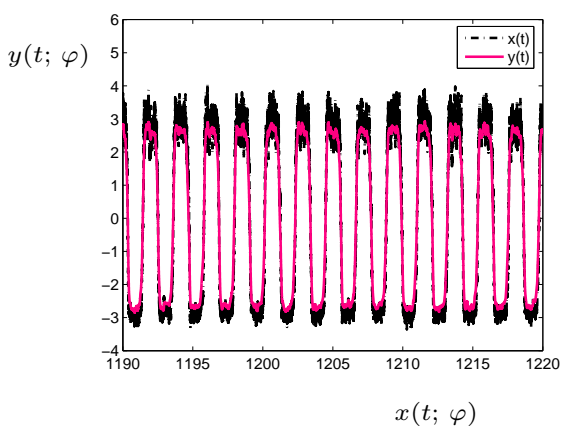

(c)

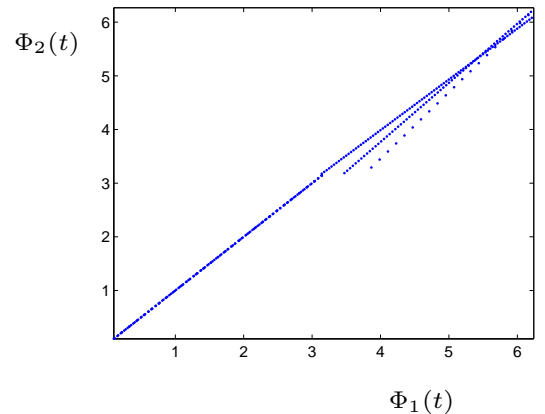

(b)

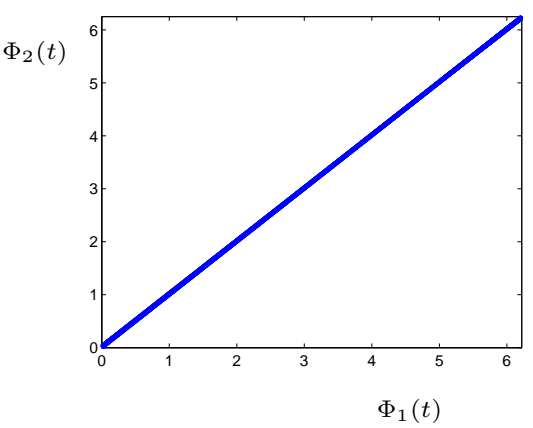

$(d)$

Figure 5. The stochastic processes defined by system (30) appears disorder when we choose $\sigma_{0}=0.05$ and $\sigma_{1}=0.3$. (a) The stochastic process $y(t ; \varphi)$ versus $x(t ; \varphi)$ at $\tau=0.38$; (b) The phase portrait of $\Phi_{2}(t)$ versus $\Phi_{1}(t)$ at $\tau=0.38$; (c) The stochastic process $y(t ; \varphi)$ versus $x(t ; \varphi)$ at $\tau=13.78$; (d) The phase portrait of $\Phi_{2}(t)$ versus $\Phi_{1}(t)$ at $\tau=13.78$.

As shown in Figure 5 (b) and (d), the stochastic processes $x(t, \varphi)$ and $y(t, \varphi)$ are always keeping in phase synchronizing states, with delay values $\tau=0.38$ and $\tau=13.78$, while we choose $\sigma_{0}=0.05$ and $\sigma_{1}=0.3$, respectively.

To find what the periodic oscillatory solution looks like with the noise perturbation, we draw the stochastic processes defined by equation (30) by taking $\tau=0.48,2.98$ and $\tau=13.78$, respectively. As shown in Figure 6, with a strong noise perturbation $\sigma_{0}=0.3$, the stochastic processes defined by equation (30) perturb a little bit around the periodic solution as in Figure 4 (a) and (b). If we take $\tau=12.48$, the synchronizing periodic solution as shown in Figure 4 (c) has a significant change and becomes non-synchronizing, although a weaker noise perturbation $\sigma_{0}=0.05$ is considered. As shown in Figure 7 (a) and (b), oscillatory solutions with a random perturbation are observed with $\sigma_{1}=0.05$ and $\sigma_{1}=0.3$, respectively. 


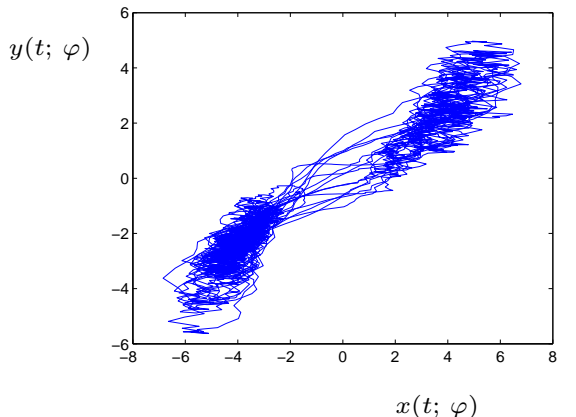

(a)

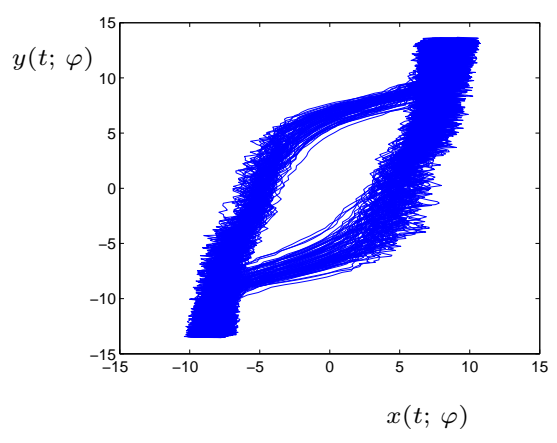

(b)

FIGURE 6. Oscillatory periodic solutions with random perturbation defined by system (30) when $\sigma_{0}=0.3$ and $\sigma_{1}=0.3$, and (a) with $\tau=0.48$; (b) with $\tau=2.98$.

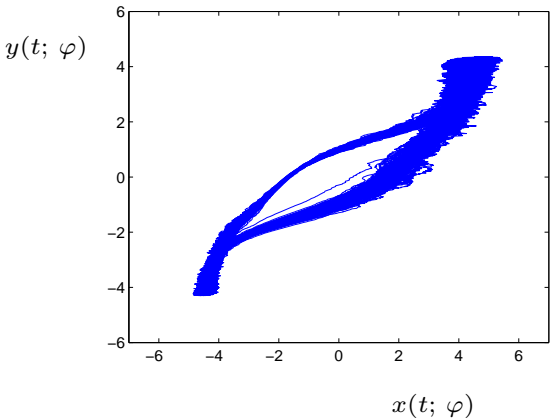

(a)

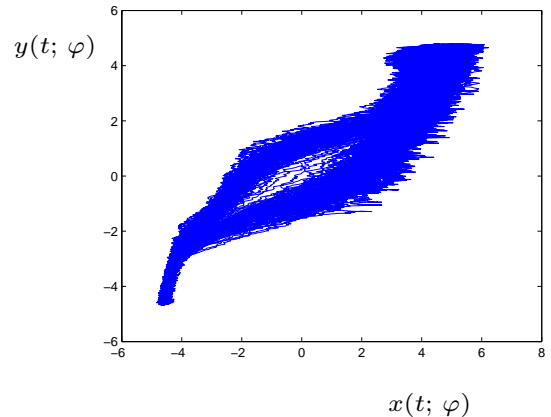

(b)

FiguRE 7. Oscillatory periodic solutions with random perturbation defined by system (30) when $\tau=13.78$, and (a) $\sigma_{0}=0.05$ and $\sigma_{1}=0.3 ;$ (b) $\sigma_{0}=0.3$ and $\sigma_{1}=0.3$.

Based on the above discussions, we can see that noise acts as an important and sensitive role in some biological systems since it is the internal natural factor. $\mathrm{Nu}$ merically, noise can induce phase synchronizing oscillatory solutions if the original state is asymptotically stable to zero. Noise can also destroy original synchronizing periodic solutions to become other type periodic oscillations.

\section{Conclusion}

In nature, the population size of many species is randomly varying with many factors. In this paper, we studied the dynamical behavior of a species which inhabits two independent patches and a nonlinear transfer rate $D e^{-p \tau}$ between two patches was assumed. The noise effect was considered on one patch and the stochastic delay model of a simple species inhabiting two patches was proposed. Without noise, we developed a geometrical method to effectively investigate stability conditions of the 
trivial solution. The stable region and the Hopf bifurcation curves were well plotted in the $(\tau-p)$ plane. Moreover, an approximate equation on the center manifold was constructed, and the Poincaré normal form with the Poincaré-Lyapunov constants was determined. With the noise effect, the first moment stability of the stochastic process was assured in the stable region. Numerically, both phase portraits of system (1) with and without noise were plotted. Synchronizing periodic solutions were found near the Hopf bifurcation values without noise, which appear to oscillate furiously when the noise effect was taken into account. On the other hand, from simulations it seems that the noise effect induced phase synchronization oscillatory solutions of system (1).

In a forthcoming paper, the noise effect will be considered on each patch and the stochastic delay model for the two-patch mode of a simple species will be studied. Synchronizing periodic solutions and oscillatory solutions will be presented both theoretically and numerically.

\section{Acknowledgments}

Part of main contents of this paper was also announced at the SIAM Conference on Analysis of Partial Differential Equations (PD07), Phoenix, Arizona, December 10-12, 2007 (http://www.siam.org/meetings/pd07/index.php). The work is partly supported by CRA (Chinese Agricultural University) Doctorate Grant 2006064 and UTPA Faculty Research Council Grant 119100.

\section{References}

[1] J. D. Murray, Mathematical Biology, Springer-Verlag, New York, 1993.

[2] Y. Kuang, Delay Differential Equations with Applications in Population Dynamics, Academic Press, Boston, 1993.

[3] W. Wischert, A. Wunderlin, A. Pelster, M. Olivier and J. Groslambert, Delay induced instabilities in nonlinear feedback systems, Phys. Rev. E 49 (1994), 203-214.

[4] K. Miyakawa and K. Yamada, Entrainment in coupled salt-water oscillators, Phys. D 127 (1999), 177-186.

[5] M. E. Starzak, Mathematical Methods in Chemistry and Physics, Springer, New York, 1989.

[6] L. Wang and X. Zou, Stability and bifurcation of bidirectional associative memory neural networks with delayed self-feedback, Internat. J. Bifur. Chaos Appl. Sci. Engrg. 15 (2005), $2145-2159$.

[7] J. D. Murray, Mathematical Biology, Springer-Verlag, New York, 2004.

[8] W. G. Aiello and H. I. Freedman, A time-delay model of single-species growth with stage structure, Math. Biosci. 101 (1990), 139-153.

[9] W. G. Aiello, H. I. Freedman and J. Wu, Analysis of a model representing stage-structured population growth with state-dependent time delay, SIAM J. Appl. Math. 52 (1992), 855-869.

[10] Y. Kuang and J. W. H. So, Analysis of a delayed two-stage population model with spacelimited recruitment, SIAM J. Appl. Math. 55 (1995), 1675-1696.

[11] E. Beretta and Y. Kuang, Geometrical stability switch criteria delay differential systems with delay dependent parameters, SIAM J. Math. Anal. 33 (2002), 1144-1165.

[12] S. A. Gourley and Y. Kuang, A stage structured predator-prey model and its dependence on maturation delay and death rate, J. Math. Biol. 49 (2004), 188-200.

[13] K. L. Cooke and P. van den Driessche, On zeros of some transcendental equations, Funkcial EKvac. 29 (1986), 77-90.

[14] K. L. Cooke, P. van den Driessche and X. Zou, Interaction of maturation delay and nonlinear birth in population and epidemic models, J. Math. Biol. 39 (1999), 332-352.

[15] S. Q. Ma, Q. S. Lu and S. L. Mei, Dynamics of a logistic population model with maturation delay and nonlinear birth rate, Discrete Contin. Dyn. Syst. Ser. B 5 (2005), 735-752. 
[16] J. Z. Lei and M. C. Mackey, Stochastic differential delay equation, moment stability, and application to hemropoietic stem cell regulation system, SIAM J. Appl. Math. 67 (2007), 387-407.

[17] Y. Qu and J. J. Wei, Bifurcation analysis in a time-delay model for prey-predator growth with stage-structure, Nonlin. Dyn. 49 (2007), 285-294.

[18] S. Petrovskii, H. Malchow and B. L. li, An exact solution of a diffusive predator-prey system, Proc. R. Soc. A 461 (2005), 1029-1053.

[19] S. B. Hsu, T. W. Hwang and Y. Kuang, Global analysis of the MichaelisMenten ratiodependent predator-prey system, J. Math. Biol. 42 (2001), 489-506.

[20] A. Longtin, Effects of noise on nonlinear dynamics, in Nonlinear Dynamics in Physiology and Medicine (A. Beuter, L. Glass, M. C. Mackey, and M. S. Titcombe, eds.), pp. 149-189, Springer-Verlag, New York, 2003.

[21] K. Burrage, P. M. Burrage and T. Tian, Numerical methods for strong solutions of stochastic differential equations: an overview, Proc. R. Soc. Lond. A 460 (2004), 373-402.

[22] B. Øksendal, Stochastic Differential Equations: An Introduction with Applications, 6th ed., Springer-Verlag, New York, 2003.

Department of Mathematics, China Agricultural University, Beijing 100083, China

Department of Mathematics, University of Texas-Pan American, Edinburg, TX 78539, USA

E-mail address: zsfeng@utpa.edu

Department of Mathematics, Beijing University of Aeronautics and Astronautics, BeIJing 100083, China 